\title{
Far-infrared polarimetry from the Stratospheric Observatory for Infrared Astronomy
}

John E Vaillancourt, David T. Chuss, Richard M. Crutcher, Jessie L. Dotson, C. Darren Dowell, et al.

John E Vaillancourt, David T. Chuss, Richard M. Crutcher, Jessie L. Dotson, C. Darren Dowell, D. Al Harper, Roger H. Hildebrand, Terry J. Jones, Alexandre Lazarian, Giles Novak, Michael W. Werner, "Far-infrared polarimetry from the Stratospheric Observatory for Infrared Astronomy," Proc. SPIE 6678, Infrared Spaceborne Remote Sensing and Instrumentation XV, 66780D (26 September 2007); doi: 10.1117/12.730922

Event: Optical Engineering + Applications, 2007, San Diego, California, United States 


\title{
Far-infrared polarimetry from the Stratospheric Observatory for Infrared Astronomy
}

\author{
John E. Vaillancourt ${ }^{a}$, David T. Chuss ${ }^{b}$, Richard M. Crutcher ${ }^{c}$, Jessie L. Dotson ${ }^{d}$, \\ C. Darren Dowell ${ }^{a, e}$, D. Al Harper ${ }^{f}$, Roger H. Hildebrand ${ }^{f}$, Terry J. Jones ${ }^{g}$, \\ Alexandre Lazarian ${ }^{h}$, Giles Novak ${ }^{i}$, and Michael W. Werner ${ }^{e}$ \\ ${ }^{a}$ Physics Department, California Institute of Technology, Pasadena, CA 91125, USA; \\ ${ }^{b}$ NASA Goddard Space Flight Center, Greenbelt, MD 20771, USA; \\ ${ }^{c}$ Department of Astronomy, University of Illinois, Urbana, IL 61801, USA; \\ $d$ NASA Ames Research Center, Moffett Field, CA 94035, USA; \\ e Jet Propulsion Laboratory, Pasadena, CA 91109, USA; \\ fDepartment of Astronomy \& Astrophysics, University of Chicago, Chicago, IL 60637, USA; \\ ${ }^{g}$ Department of Astronomy, University of Minnesota, Minneapolis, MN 55455, USA; \\ ${ }^{h}$ Department of Astronomy, University of Wisconsin, Madison, WI 53706, USA; \\ ${ }^{i}$ Department of Physics \& Astronomy, Northwestern University, Evanston, IL 60208, USA
}

\begin{abstract}
Multi-wavelength imaging polarimetry at far-infrared wavelengths has proven to be an excellent tool for studying the physical properties of dust, molecular clouds, and magnetic fields in the interstellar medium. Although these wavelengths are only observable from airborne or space-based platforms, no first-generation instrument for the Stratospheric Observatory for Infrared Astronomy (SOFIA) is presently designed with polarimetric capabilities. We study several options for upgrading the High-resolution Airborne Wideband Camera (HAWC) to a sensitive FIR polarimeter. HAWC is a $12 \times 32$ pixel bolometer camera designed to cover the $53-215 \mu \mathrm{m}$ spectral range in 4 colors, all at diffraction-limited resolution $(5-21$ arcsec). Upgrade options include: (1) an external set of optics which modulates the polarization state of the incoming radiation before entering the cryostat window; (2) internal polarizing optics; and (3) a replacement of the current detector array with two state-of-the-art superconducting bolometer arrays, an upgrade of the HAWC camera as well as polarimeter. We discuss a range of science studies which will be possible with these upgrades including magnetic fields in star-forming regions and galaxies and the wavelength-dependence of polarization.
\end{abstract}

Keywords: polarimetry, far-infrared astronomy, instrumentation, interstellar medium, magnetic fields, dust, airborne astronomy, SOFIA

\section{INTRODUCTION}

Mapping polarimetry at far-infrared/submillimeter wavelengths began on the Kuiper Airborne Observatory (KAO) more than 20 years ago. Over the course of the KAO's lifetime polarimetric instruments evolved from single pixel devices ${ }^{1}$ to larger arrays ${ }^{2}$ and eventually moved to ground-based observatories. ${ }^{3,4}$ Far-infrared (FIR) and submillimeter observations of dense molecular clouds, protostellar disks and envelopes, the Galactic center, and one external galaxy have shown that dust emission from these objects is polarized (at levels of $\sim 2-10 \%$ ) at almost every point. ${ }^{5-7}$ The alignment of dust grains with the local magnetic field ${ }^{8}$ allows one to infer the direction of the magnetic field projected onto the plane of the sky. This allows studies of magnetic fields' interactions with the local interstellar medium and their role in star and galaxy formation ${ }^{9-12}$ as well as studies of dust and cloud properties. ${ }^{13,14}$

As successor to the KAO, the Stratospheric Observatory for Infrared Astronomy (SOFIA) offers a new frontier for observations in the infrared-to-submillimeter spectral regime. Unfortunately, no first-light SOFIA

Further author information: J.E.V.: E-mail: johnv@submm.caltech.edu

Infrared Spaceborne Remote Sensing and Instrumentation XV, edited by Marija Strojnik-Scholl, Proc. of SPIE Vol. 6678, 66780D, (2007) · 0277-786X/07/\$18 - doi: 10.1117/12.730922 


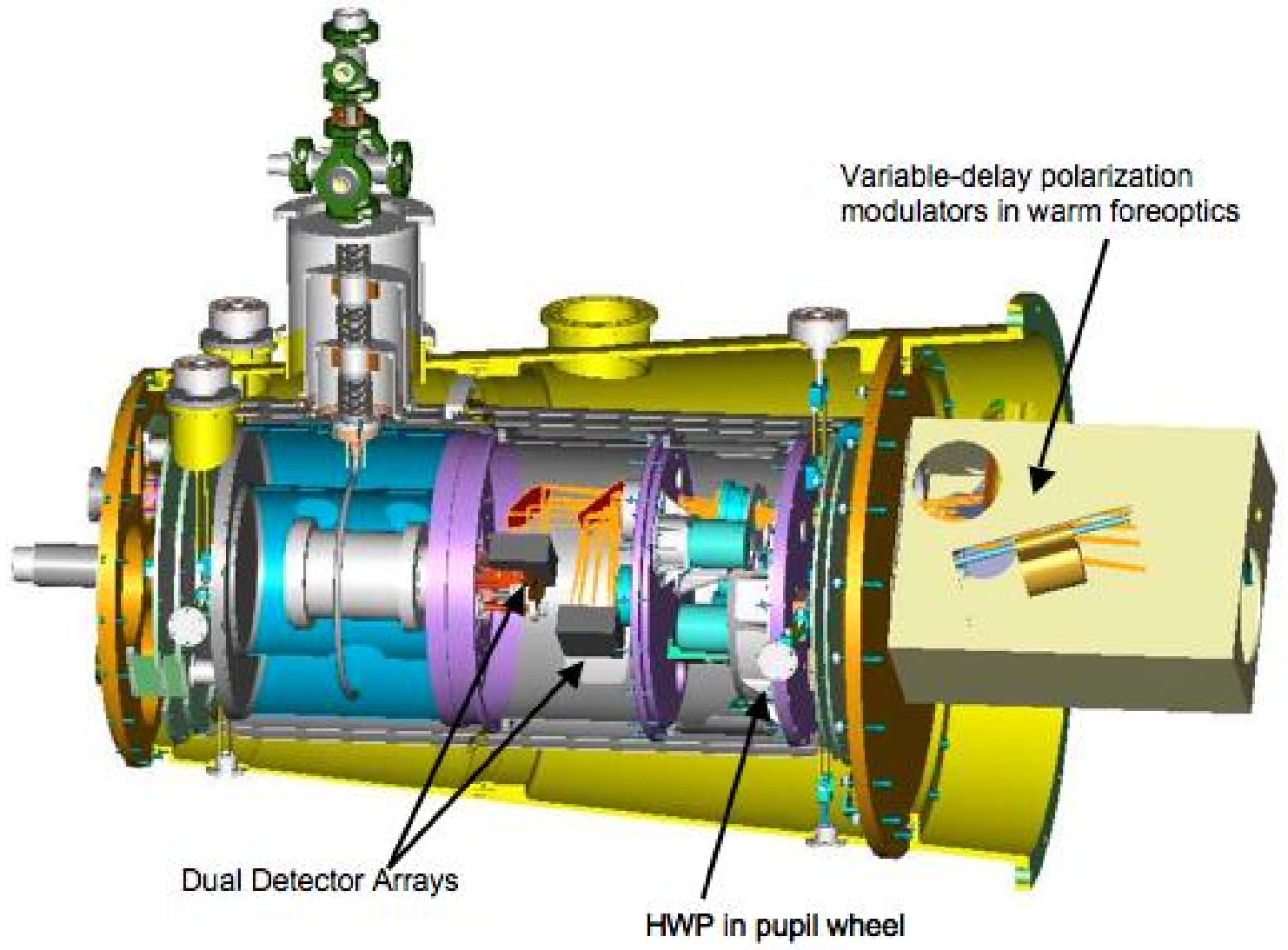

Figure 1. The HAWC cryostat showing the locations of several proposed polarimetric upgrades: dual-polarization detector arrays, wave-plates (HWP), and the variable-delay modulator.

instruments ${ }^{15}$ have existing capability for accurate polarimetry. Both a far-infrared $(\text { Hale })^{16}$ and mid-infrared ${ }^{17}$ polarimeter have been proposed as future SOFIA instruments. Here we discuss three options for upgrading the High-resolution Airborne Wideband Camera (HAWC) ${ }^{18}$ to a sensitive FIR polarimeter.

HAWC is a first-light instrument for SOFIA. It consists of a $12 \times 32$ pixel bolometer camera designed to cover the $53-215 \mu \mathrm{m}$ spectral range in 4 colors, all at diffraction-limited resolution $\left(5^{\prime \prime}-21^{\prime \prime}\right)$. A complete upgrade of the HAWC camera into a polarimeter (which we call Hale) will include replacing the existing 384 pixel array of silicon bolometers with two superconducting bolometer arrays. Each such array would consist of over 5000 pixels and detect orthogonal polarization components selected by wire grids close to the detectors. A cold $(4 \mathrm{~K})$ crystal half-wave plate (HWP) upstream from the grids modulates the incoming radiation. In this way we create a sensitive, diffraction-limited, 4-color polarimeter. Additionally, the dual-polarization measurement and the detector upgrade yield a photometer with a much larger field-of-view than the original HAWC instrument.

We consider two other options (called HAWC-pol) as "pathfinder" programs working towards the more complete Hale instrument. These options are attractive as it is feasible that they may be incorporated into HAWC soon after its first light on SOFIA. In both HAWC-pol options we retain the existing $12 \times 32$ silicon bolometer array and measure only a single component of polarization. Option (1) places one or more rotating HWPs at the cold HAWC pupil stop, followed by a polarizing grid. In option (2) we place only a polarizing grid at the pupil and modulate the polarization outside the cryostat using a variable-delay polarization modulator, or polarization "switch". Details of the upgrade components are discussed in Sect. 3; their locations in the HAWC cryostat are shown in Fig. 1. The technical specifications for both Hale and HAWC-pol are shown in Table 1. 
Table 1. HAWC-pol and Hale specifications.

\begin{tabular}{|c|c|c|c|c|}
\hline Parameter & Band 1 & Band 2 & Band 3 & Band 4 \\
\hline Central Wavelength $(\mu \mathrm{m})$ & 53 & 89 & 155 & 216 \\
\hline Bandwidth FWHM $(\Delta \lambda / \lambda)$ & 0.16 & 0.19 & 0.22 & 0.21 \\
\hline Pixel Size (arcsec) & 2.3 & 3.5 & 6.0 & 8.0 \\
\hline Resolution FWHM (arcsec) & 5.4 & 9.0 & 16 & 22 \\
\hline Field of view, HAWC-pol (arcmin) & $0.5 \times 1.2$ & $0.7 \times 1.9$ & $1.2 \times 3.2$ & $1.6 \times 4.3$ \\
\hline Field of view, Hale (arcmin) & $2.4 \times 3.0$ & $3.7 \times 4.7$ & $6.4 \times 8.0$ & $8.5 \times 8.5$ \\
\hline Background Power (pW/pixel) & 24 & 20 & 20 & 10 \\
\hline $\mathrm{NEP}^{a}\left(\mathrm{fW} \mathrm{Hz}{ }^{-1 / 2}\right)$ & 0.43 & 0.30 & 0.24 & 0.14 \\
\hline $\operatorname{NEFD}^{b}\left(\mathrm{Jy} \mathrm{s}^{1 / 2}\right)$ & 0.87 & 0.59 & 0.60 & 0.44 \\
\hline Polarization uncertainty, ${ }^{c}$ HAWC-pol (\%) & 0.26 & 0.18 & 0.18 & 0.13 \\
\hline Polarization uncertainty, ${ }^{c}$ Hale $(\%)$ & 0.18 & 0.13 & 0.13 & 0.09 \\
\hline Position angle uncertainty $(P=3 \%),{ }^{c}$ HAWC-pol (degrees) & 2.5 & 1.7 & 1.7 & 1.3 \\
\hline Position angle uncertainty $(P=3 \%),{ }^{c}$ Hale (degrees) & 1.7 & 1.2 & 1.2 & 0.9 \\
\hline
\end{tabular}

\section{SCIENTIFIC GOALS}

\subsection{Turbulent Star Formation}

The paradigm for star formation, once seen as depending on slow diffusion of magnetic fields out of cloud cores, has shifted to a violent one,${ }^{19}$ the dynamics of which are governed by magnetic compressible turbulence. ${ }^{20,21}$

A unique view of the turbulent field is provided by polarimetry of emission from magnetically aligned grains. Problems of interpreting the statistics of $2 \mathrm{D}$ polarization maps in terms of the underlying statistics of the 3D magnetic fields are becoming tractable with modern theoretical work on radiative transfer, grain alignment, and turbulence, even in the case of inhomogeneous clouds containing dense clumps and clusters of embedded stars. ${ }^{22}$ The problem of field bending due to large-scale non-turbulent effects is being addressed by choosing reference systems along the local projected direction of the field rather than the mean field for the whole cloud.

When maps of polarized emission can be made with SOFIA's resolution, dynamic range, and spectral coverage, it will be feasible, not only to test predictions of turbulent vs. static star formation, but also to determine characteristics of the magnetic fields such as the ratio of fluctuating to mean components, the energy spectrum, and the distribution of magnetic field strengths.

\subsection{Dust Grains and the Polarization Spectrum}

Recent years have been marked by substantial progress in the understanding of grain alignment. ${ }^{23}$ Research on radiative torques ${ }^{24-27}$ and grain dynamics ${ }^{28-35}$ have made it possible to explain the otherwise perplexing results of FIR polarization from starless $\operatorname{cores}^{36}$ and to make quantitative predictions of grain alignment along lines of sight through dense clouds.

The anisotropic radiation responsible for aligning grains is ubiquitous in astrophysics. Thus polarized emission from dust grains can trace magnetic fields in various astrophysical environments. (While radiative torques provide the aligning mechanism, the alignment occurs with respect to the local magnetic field.) Features in the measured polarization spectra of molecular clouds (Fig. 2) have been attributed to varying dust temperatures and polarizing efficiencies along the line-of-sight. ${ }^{13,37,38}$ The alignment of grains, their temperature, and their emission properties depend on the radiation field to which they are exposed. As a result, multi-wavelength polarimetry can be used for detailed studies of magnetic fields even within unresolved objects (e.g. Sect. 2.3; Fig. 2).

A polarimeter on SOFIA will make it possible to pursue these studies with more passbands, more pixels, and improved spatial resolution. 

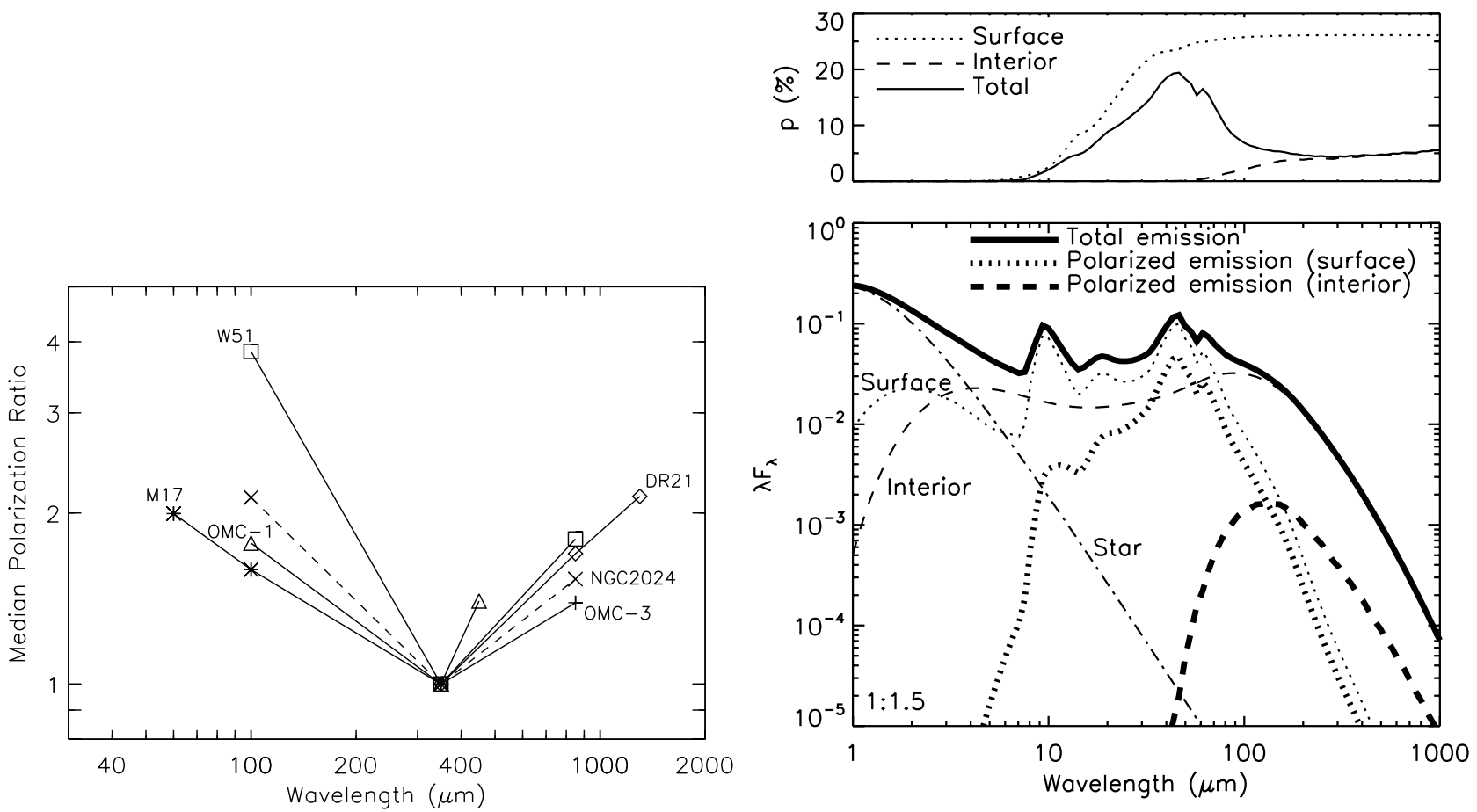

Figure 2. Left: Measured polarization spectra of several molecular cloud envelopes, each cloud is normalized at $350 \mu \mathrm{m} .{ }^{38,39}$ Right: Predicted spectral energy distribution (bottom) and polarization spectrum (top) for protoplanetary disk emission. ${ }^{40}$ Note that the peak in the polarization occurs at $45 \mu \mathrm{m}$, corresponding approximately to the shortest HAWC passband.

\subsection{Polarization spectra of protoplanetary disks}

By the time a young stellar object reaches the Class II stage, a.k.a. T-Tauri star (TTS) stage, the star has acquired approximately its final mass, but a residual disk still contains diffuse matter that will presumably be incorporated into planets. According to some current theories of planet formation, ${ }^{41}$ the first step is for the grains in such a protoplanetary disk to coagulate, forming particles with sizes of order one millimeter or larger. Observational evidence of such "large grains" has been obtained via modeling of the submillimeter-millimeter spectral energy distributions of TTS disks. ${ }^{42-45}$ If these models are correct, then the optical depths for absorption and scattering will approach or exceed unity across much of the FIR-to-millimeter spectrum. ${ }^{43}$ In this case, the polarization of the disk thermal emission will likely contain contributions from selective absorption and scattering. ${ }^{46}$ Although this complicates the interpretation, it also presents us with a new opportunity: Because the cross-sections for absorption and scattering, and thus the polarization characteristics, depend strongly on grain size for sizes comparable to the wavelength, we may be able to confirm the existence of millimeter-sized grains and further constrain the grain size via polarimetry of the disk emission.

Polarimetry of protoplanetary disk emission has been sparse and the modeling is at an early stage, but with the advent of SOFIA, ALMA*, and EVLA ${ }^{47}$ it will become possible to carry out sensitive polarimetry across the FIR-to-millimeter wavebands for a sample of bright TTS disks. A recent model ${ }^{40}$ shows that the polarization characteristics depend strongly on grain size (Fig. 2). Initial polarimetric observations of TTS disk emission, obtained at the James Clerk Maxwell Telescope ${ }^{48}$ and at the Caltech Submillimeter Observatory (M. Krejny, priv. comm.) suggest polarization levels of one to several percent. Four-color polarimetry with Hale or HAWCpol will place constraints on grain size and provide information on the magnetic fields (the latter of which may influence disk evolution ${ }^{49}$ ).

\footnotetext{
*http://www.alma.nrao.edu/
} 


\subsection{Connecting Large- and Small-Scale Interstellar Fields}

The role played by magnetic fields in star formation has remained uncertain. At one extreme of the theories of star formation is that magnetic fields control the formation and evolution of the molecular clouds from which stars form, including the formation of cores and their gravitational collapse to form protostars. ${ }^{50}$ The other extreme is that magnetic fields are unimportant, with molecular clouds forming at the intersection of turbulent supersonic flows in the interstellar medium. ${ }^{51}$ In spite of considerable effort to observe magnetic fields in molecular clouds in order to resolve this uncertainty, the issue remains controversial. SOFIA with Hale or HAWC-pol will provide a crucial link between large- and small-scale data on interstellar magnetic fields that will be essential to understand the role of magnetic fields in star formation.

On the largest scales, observations of stellar polarization ${ }^{52,53}$ and Planck $^{54}$ polarization maps at $5^{\prime}$ resolution enable mapping of the Galactic to molecular cloud scale structure of the interstellar magnetic field. HAWC-pol will make it possible to study structure of cores relative to large-scale magnetic fields. Some predicted phenomena that require the correlation of the large- and small-scale maps of magnetic field structures include collapse of mass along field lines to form cores flattened along field lines, hourglass morphology fields in cores with the core fields connecting to the larger scale molecular cloud fields, magnetic braking of cores that will twist the fields as angular momentum is transferred outward from cores to envelopes, and bipolar outflows from protostars with magnetic fields being parallel to the outflows.

On the smallest scales ( $<$ few arcseconds), ALMA will image protostars, protoplanetary disks, and the inner parts of protostellar outflows. In spite of the much larger telescope collecting area of ALMA, the shorter wavelengths and much higher bandwidths observed by HAWC mean that HAWC-pol will have comparable sensitivity to ALMA for polarization mapping of extended emission. Theoretical models predict the morphology of the connection of magnetic fields in these very small scale phenomena to the larger scale fields in the cores from which protostars form. Combining ALMA and HAWC-pol maps will probe the full spatial structure of these phenomena and allow testing of the star formation theoretical results.

\subsection{The Galactic Center and External Galaxies}

Our proximity to the Galactic Center provides an opportunity to study the physics of galactic nuclei in great detail. There is evidence that magnetic fields play an important role in the dynamics of the Galactic center region as evidenced by the prominent Radio $\mathrm{Arc}^{55}$ and other non-thermal filaments ${ }^{56,57}$ that exist in this region. These filaments are thought to be the result of relativistic electrons spiraling along lines of magnetic flux, and though the brightest are oriented perpendicular to the plane, more recently discovered filaments have been found with other orientations. Submillimeter polarimetry has indicated that the large-scale magnetic field in the molecular gas is generally parallel to the plane ${ }^{58}$ in stark contrast to the brightest radio filaments. However, on smaller scales the field has been found to be more complex (Fig. 3). ${ }^{10}$

Key outstanding questions concerning the magnetic fields in the Galactic center are: (1) What is the geometry of the field, (2) what is the strength of the field, and (3) how do the radio filaments form? The multi-frequency capability of a HAWC polarimeter will allow us to separately measure the field in different components along the line-of-sight. This is particularly important in the Galactic center, as the region encompasses a wide range of temperatures. In addition, the high angular resolution may enable one to probe the interaction sites between filaments and associated molecular clouds. Such interactions may provide a mechanism for electron acceleration which is required for the formation of the filaments. This has the potential for providing estimates of the local magnetic field strength and constraining models for filament formation.

External galaxies give us the opportunity to study the magnetic field geometry of an entire system viewed from the outside. Edge on spiral galaxies in particular will allow us to sample the integrated interstellar medium along a number of lines of sight, weighted of course by the distribution of dust, stars, and heating radiation as a function of galactocentric radius. For many galaxies such as NGC 891, NGC 4565, etc., the edge-on disk is only about one beam wide at $60 \mu \mathrm{m}$ and spans many beam widths across the sky. Comparison of very active galaxies such as M82, moderately active disks such as in NGC 891 and more quiescent disks such as in NGC 4565 will allow Hale to find and distinguish large-scale structures in the magnetic field deep within these galaxies. These features could include massive blowouts, for example, with a projected magnetic field geometry vertical to the galactic plane, undetectable with optical and near-infrared polarimetry. 


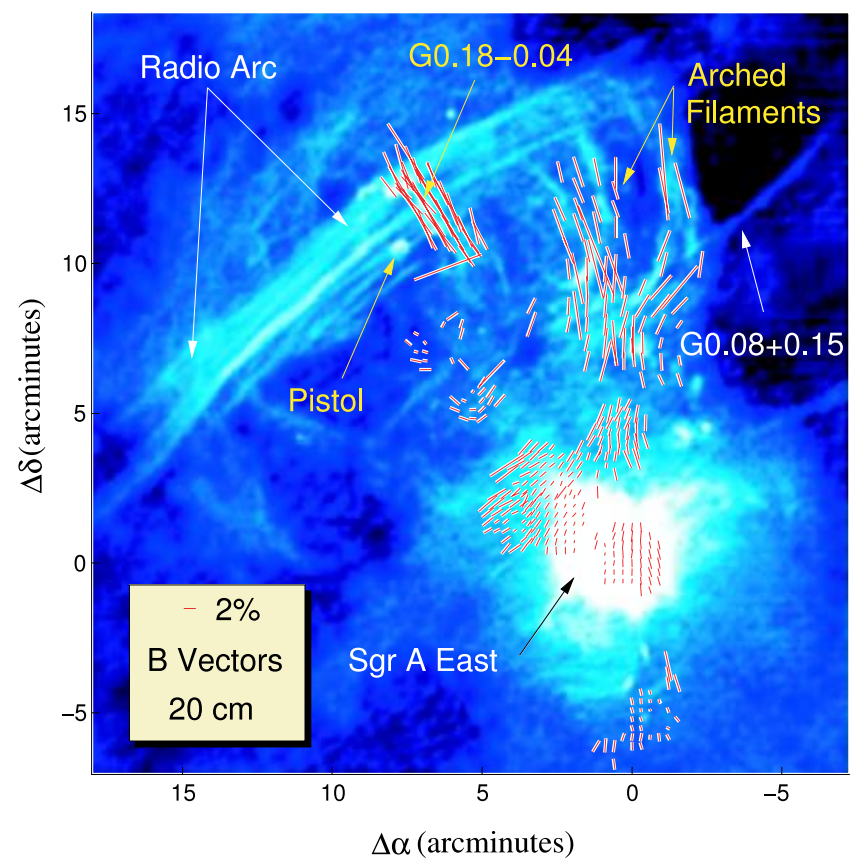

Figure 3. Polarization and radio map of the Galactic center. ${ }^{10}$ Inferred magnetic field vectors from FIR/submillimeter polarimetry are superposed on a $20 \mathrm{~cm}$ continuum VLA image. The $100 \mu \mathrm{m}$ vectors appear to trace the thermal arched filaments while the magnetic field in the molecular cloud associated with G0.18-0.04 (350 $\mu$ m polarimetry) is perpendicular to the field traced by the non-thermal filaments of the Radio Arc.

\section{INSTRUMENT DESIGN}

\subsection{Polarization Modulation}

Atmospheric constraints on suborbital FIR/submillimeter polarimetry have been described in the literature. ${ }^{59,60}$ The two main effects are: 1) the temporal change of the atmospheric transmission, and 2) "sky noise", which is the spatially and temporally variable emission from the atmosphere which is not completely eliminated by techniques such as chopping. Both effects can be mitigated by a polarimeter which observes the field of view in two polarizations simultaneously. ${ }^{60}$ However, it is often not practical to implement the dual-polarization approach in an existing instrument design. An alternate solution is to perform polarization modulation quickly, so as to "freeze" the atmosphere. ${ }^{59,61}$ Both the dual-polarization and rapid-polarization-modulation techniques work because atmospheric emission is intrinsically unpolarized.

For HAWC-pol, we are considering two approaches to rapid polarization modulation - a variable-delay polarization modulator (VPM), and a continuously-spinning half-wave plate (HWP). Both devices are considered in more detail in the following sections. However, several aspects of the design are common.

In our observing plan, the chopping secondary mirror of SOFIA is the fastest modulation, and the VPM or HWP is a slower modulation. Secondary chopping is ideally done at a suitably high frequency where the noise of the difference is dominated by white noise from the photon arrival statistics. ${ }^{62}$ The typical atmospheric noise spectrum for SOFIA FIR observations is not yet known, but the mechanical capabilities support chopping at 5 $-20 \mathrm{~Hz}$, which we expect will be fast enough in most conditions.

Although the atmospheric sky noise is intrinsically unpolarized, it will become partially polarized by nonnormal reflections in the optics, in particular the dichroic tertiary mirror. Past experience with this type of mirror $^{5}$ indicates that dichroic mirror polarization will be $\sim 1 \%$. Therefore, the sky noise will be registered as a polarization noise at a level $\sim 100 \times$ reduced. Since the polarization modulation is at a lower frequency, the frequency spectrum of the atmospheric noise matters. If the noise spectrum is proportional to $1 / f^{2}$ (in amplitude units), then the polarization modulation needs to be at a frequency at least as large as $\sim 0.1 \times$ the requirement 

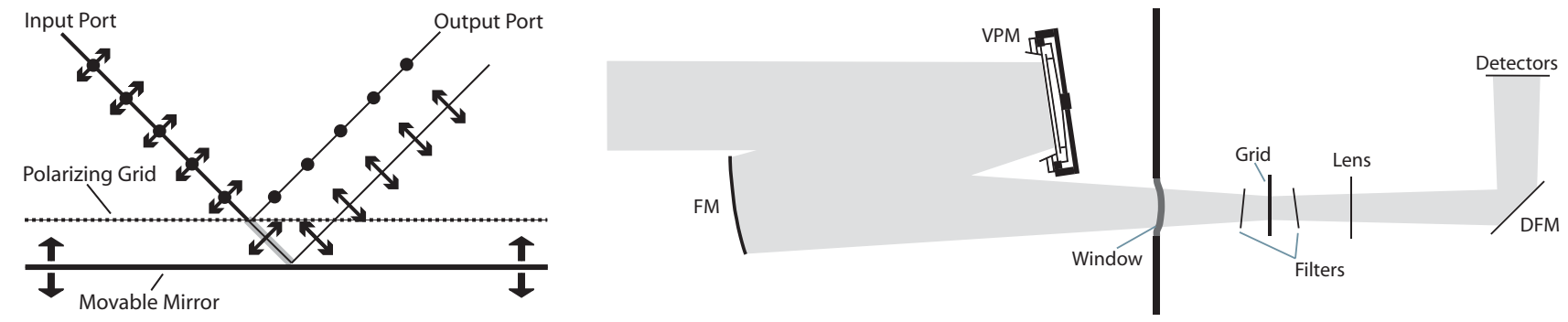

Figure 4. Left: A schematic for the Variable-delay Polarization Modulator (VPM). A controlled, variable delay (shaded line) can be introduced between two orthogonal linear polarizations. Right: The current HAWC fore-optics can be modified for the polarimeter. The optics to the left of the window are at the ambient temperature; these include the VPM and the Focusing Mirror (FM). The optics to the right of the window are all $\sim 4 \mathrm{~K}$; these include the analyzing grid, filters, lens, detector folding mirror (DFM), and the detectors themselves.

for the chopping. For HAWC-pol, we plan on polarization modulation at $\sim 1 \mathrm{~Hz}$ with either a square-wave profile (VPM) or sinusoidal profile (HWP or VPM).

Since all of the radiation from the atmosphere is partially polarized by the dichroic mirror, continuous polarization modulation will produce a large, nearly constant periodic signal on the detectors equivalent to a $\sim 1 \mathrm{~K}$ (Rayleigh-Jeans) load. The detector readout must have a dynamic range which accepts this signal, and the linearity of the detector over this range must be understood. Since the polarization modulation is used with faster spatial modulation, the celestial polarization signal will appear at $\sim 1 \mathrm{~Hz}$ "sidebands" of the chopping frequency and its harmonics; the detector readout must have good noise performance in this frequency range. All of these requirements are met by the HAWC system. The large $1 \mathrm{~Hz}$ atmospheric signal can be filtered out in an early data analysis step.

\subsection{A Continuously-Spinning Half-Wave Plate}

In this section, we describe an approach to HAWC polarimetry using a uniformly rotating birefringent quartz HWP. Cryogenic crystal quartz has well characterized o-ray and e-ray indices of refraction, and thin waveplates should have loss $<5 \%$ over most of the FIR. ${ }^{63,64}$ Although waveplates can be stacked to achieve nearachromaticity over an octave of wavelength, ${ }^{4}$ the HAWC filter bands cover two octaves. We expect that at least two HWPs will be required and perhaps four.

We have identified the HAWC pupil wheel as an ideal location to locate polarimeter HWPs. An existing mechanism provides infrequent selection of up to eight pupil apertures for diagnostic purposes. In practice, no more than four of these apertures are needed for the camera, ${ }^{18}$ leaving four positions available for HWPs. The pupil wheel is located at an image of the SOFIA primary mirror, which is an optimum location for a HWP. ${ }^{65}$ The pupil image has a diameter of $38 \mathrm{~mm}$, a manageable size for quartz elements. A wire-grid polarizer will be located behind each HWP to provide polarization detection. The polarimeter is removed from the optical path by rotating the pupil wheel to select the camera-mode pupil aperture.

For $1 \mathrm{~Hz}$ polarization modulation, the HWP must rotate at $15 \mathrm{rpm}$. Even at this low speed, induced vibration and microphonic response of the high-impedance bolometer detectors is a concern. We will avoid ball bearings and gear trains in the design and will instead use jewel bearings and three rollers to suspend each half-wave plate and a motor which directly torques permanent magnets attached to the waveplate. We expect dissipation in the bearings to be under $1 \mathrm{~mW}$, which can be carried away through the pupil wheel. An optical encoder will monitor the HWP position. Implementing this polarimeter solution is straightforward and will require reconstruction of the pupil wheel, the plate which holds it, and the Geneva mechanism which defines the eight positions.

\subsection{The Variable-delay Polarization Modulator (VPM)}

In the case of the Variable-delay Polarization Modulator (VPM), polarization is modulated by allowing a controlled phase delay to be introduced between two orthogonal linear polarizations. The basic principle is shown in Fig. 4, and consists of a grid/mirror pair. Incident partially-polarized radiation comes in from the left. One 
Table 2. Measuring Polarization with a VPM

\begin{tabular}{ccc}
\hline \hline $\begin{array}{c}\text { VPM Rotation Angle } \\
\text { (degrees) }\end{array}$ & $\begin{array}{c}\Delta \phi \\
\text { (radians) }\end{array}$ & Polarization State \\
\hline 0 & $2 \pi$ & $I+Q$ \\
0 & $\pi$ & $I-Q$ \\
45 & $2 \pi$ & $I+U$ \\
45 & $\pi$ & $I-U$ \\
\hline
\end{tabular}

component of polarization is reflected from the front of a grid. The other passes through the grid to a parallel mirror and is reflected back through the grid where the two beams are recombined. The component transmitted by the grid experiences an additional path length (which is shaded in the figure). As the grid-mirror separation is modulated, the polarization measured by polarization-sensitive detectors changes in a predictable way. ${ }^{66}$ Various instruments have utilized devices with this architecture and have referred to them by various names. ${ }^{66-70}$ The nomenclature "VPM" used here emphasizes the functionality as a polarization modulator and that the degree of freedom employed is that of a variable phase delay between linear polarizations.

The polarization modulation for HAWC-pol must be flexible enough to accommodate all 4 HAWC passbands. As long as the grid is sufficiently fine for the $53 \mu \mathrm{m}$ band, we only need to change the grid-mirror separations to optimize the VPM for polarization modulation across the different bands.

By making two changes to the current HAWC configuration, we can use a single VPM to measure Stokes Q and $U$ for the four HAWC passbands: 1) Replace the warm folding flat mirror with a VPM that is mounted on a rotatable stage, and 2) Add two analyzer grids to the open slots on the aperture (pupil) wheel that have a relative orientation of $45^{\circ}$. Figure 4 also shows the warm and cold optics for HAWC with the proposed modifications for a VPM-based polarimeter.

The polarimeter will have two modes, one to measure Stokes Q in the instrument coordinate system and the other to measure Stokes U. These will be differentiated by use of the two different analyzer grids that are oriented at a $45^{\circ}$ with respect to one another. The VPM is placed on a rotator such that the relative angle of the VPM wires with respect to whichever analyzer grid is used is always $45^{\circ}$. In this way the measured signal on the detectors in each of the modes is

$$
\begin{gathered}
S_{Q}=I+Q \cos (\Delta \phi)+V \sin (\Delta \phi), \\
S_{U}=I+U \cos (\Delta \phi)+V \sin (\Delta \phi),
\end{gathered}
$$

where $\Delta \phi$ is the phase delay introduced by the VPM. This can be scaled to any HAWC frequency by changing the amplitude of the grid-mirror separation. By using half- and full-wave delays for a given band, we can use the VPM as a polarization "switch" to rapidly change the polarization state to which the detectors are sensitive from $I+Q \rightarrow I-Q$ or $I+U \rightarrow I-U$ depending upon the mode of operation. Table 2 illustrates the polarization measurement strategy.

\subsection{New Technology Large-format Detector Arrays}

To enable revolutionary FIR polarimetry, Hale will require simultaneous detection of two orthogonal linear polarizations. To accomplish this, we plan to implement two focal planes with 5,120 detectors each, using Goddard Space Flight Center's Backshort-Under-Grid (BUG) technology. ${ }^{71}$ As in the SCUBA-2 design, ${ }^{72}$ four such $32 \times 40$ subarrays will be tiled to produce each 5,120 element array.

The BUG architecture is a two-piece, keyed assembly in which a two-dimensional array of backshorts are mounted under a matching array of suspended membranes. (It should be noted here that for Hale, the term "backshort" is a misnomer because in order to enable good performance over all four passbands, we will actually employ a "back termination."). Transition-Edge Sensors (TES) are fabricated on the membranes and the leads are "wrapped around" the frame to the back of the device at which point electrical connections to the multiplexer can be made via bump-bonding. The pixel size in this architecture is $1.135 \mathrm{~mm}$ so that an array of BUGs can 

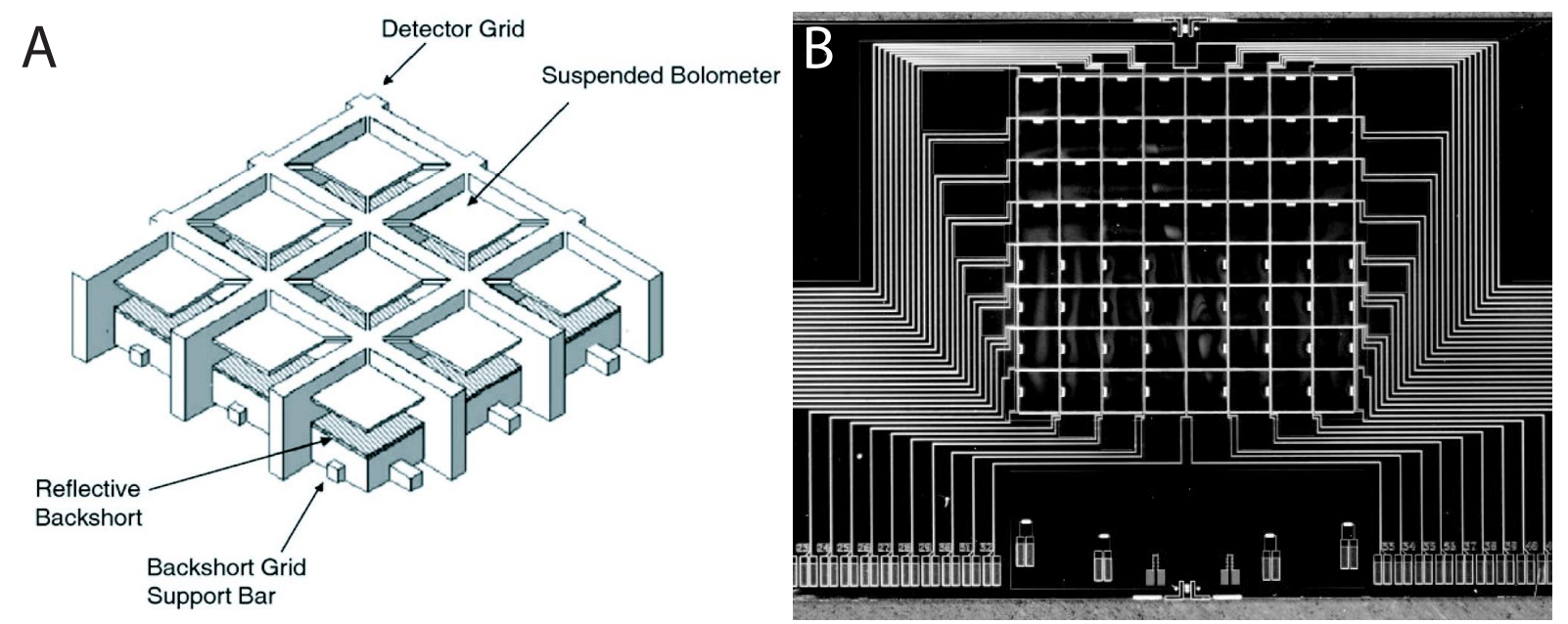

Figure 5. a) The Backshort-Under-Grid (BUGs) architecture is shown schematically. The absorber/detector membranes are suspended in a frame of silicon. Electrical and thermal connections to the frame are made over thin silicon legs. The backshort/termination assembly fits into keyed structures in the detector grid frame. Electrical connections are made to the SQUID multiplexer by wrap-around vias that are made along the grid frame. b) A prototype $8 \times 8$ detector frame is shown. For this prototype, the detector leads are fanned out in the plane of the detectors rather than wrapped around to the back of the assembly as they will be for the $32 \times 40$ arrays. $^{71}$

be mounted to the SCUBA-2 $32 \times 40$ two-dimensional time-domain SQUID multiplexer developed by NIST. ${ }^{73}$ Figure 5a shows a schematic of the BUG architecture.

The suspended silicon membranes on the front piece of the BUG assembly will be ion-implanted to achieve a surface impedance of $157 \Omega$ per square. This process has been demonstrated for the Atacama Cosmology Telescope $(\mathrm{ACT})^{74}$ detectors. The back short assembly, which for single-band implementations is made reflective, will be coated with an absorbing film to prevent unwanted resonances. This absorbing strategy is similar to that used on HAWC, and will result in a $50 \%$ efficiency across all four passbands. As in the ACT detectors, a TES consisting of a molybdenum-gold bilayer with noise-reducing normal metal bars will be fabricated at the edge of each pixel.

NASA/Goddard has developed an $8 \times 8$ pixel version of this array as a step towards the realization of kilopixel arrays. One such array is shown in Fig. 5b. In this device, the TES leads are routed on the top layer of the device to bond pads at the edge of the structure. Multiplexing is done external to the detector chip. An $8 \times 16$ version with $2 \mathrm{~mm}$ pixels has been fabricated for the GISMO (Goddard-IRAM Superconducting (2)-Millimeter Observer) Instrument. ${ }^{75}$

\section{ACKNOWLEDGMENTS}

We would like to thank Fabian Heitsch, Jungyeon Cho, Diego Falceta-Gonçalves, Megan Krejny, Jesse Wirth, Harvey Moseley, and Leslie Looney for useful discussions regarding the material in this manuscript. This work has been partially supported by NSF grants 0505124 to the University of Chicago, AST-0540882 to the California Institute of Technology, and AST-0505230 to Northwestern University. A.L. acknowledges support from the NSF Center for Magnetic Self-Organization in Laboratory and Astrophysical Plasmas and grant AST-0507164. R.M.C. acknowledges partial support from NSF grant AST-0606822.

\section{REFERENCES}

1. R. H. Hildebrand, M. Dragovan, and G. Novak, "Detection of submillimeter polarization in the Orion nebula," ApJ 284, pp. L51-L54, 1984.

2. S. R. Platt, R. H. Hildebrand, R. J. Pernic, J. A. Davidson, and G. Novak, " $100-\mu$ m array polarimetry from the Kuiper Airborne Observatory: Instrumentation, techniques, and first results," PASP 103, pp. 1193$1210,1991$. 
3. C. D. Dowell, R. H. Hildebrand, D. A. Schleuning, J. E. Vaillancourt, J. L. Dotson, G. Novak, T. Renbarger, and M. Houde, "Submillimeter array polarimetry with Hertz," ApJ 504, pp. 588-598, 1998.

4. J. S. Greaves, et al., "A submillimetre imaging polarimeter at the James Clerk Maxwell Telescope," MNRAS 340, pp. 353-361, 2003.

5. J. L. Dotson, J. Davidson, C. D. Dowell, D. A. Schleuning, and R. H. Hildebrand, "Far-infrared polarimetry of Galactic clouds from the Kuiper Airborne Observatory," ApJS 128, pp. 335-370, 2000.

6. J. L. Dotson, J. A. Davidson, C. D. Dowell, L. Kirby, J. E. Vaillancourt, and R. H. Hildebrand, "Polarimetry of bright submillimeter objects from the Caltech Submillimeter Observatory." in prep., 2007.

7. H. Li, G. S. Griffin, M. Krejny, G. Novak, R. F. Loewenstein, M. G. Newcomb, P. G. Calisse, and D. T. Chuss, "Results of SPARO 2003: Mapping magnetic fields in giant molecular clouds," ApJ 648, pp. 340-354, 2006.

8. L. J. Davis and J. L. Greenstein, "The polarization of starlight by aligned dust grains," ApJ 114, pp. 206$240,1951$.

9. D. A. Schleuning, "Far-infrared and submillimeter polarization of OMC-1: Evidence for magnetically regulated star formation," ApJ 493, pp. 811-825, 1998.

10. D. T. Chuss, J. A. Davidson, J. L. Dotson, C. D. Dowell, R. H. Hildebrand, G. Novak, and J. E. Vaillancourt, "Magnetic fields in cool clouds within the central 50 parsecs of the Galaxy," ApJ 599, pp. 1116-1128, 2003.

11. B. C. Matthews, C. D. Wilson, and J. D. Fiege, "Magnetic fields in star-forming molecular clouds. II. The depolarization effect in the OMC-3 filament of Orion A," ApJ 562, pp. 400-423, 2001.

12. J. S. Greaves, W. S. Holland, T. Jenness, and T. G. Hawarden, "Magnetic field surrounding the starburst nucleus of the galaxy M82 from polarized dust emission," Nature 404, pp. 732-733, 2000.

13. R. H. Hildebrand, J. L. Dotson, C. D. Dowell, D. A. Schleuning, and J. E. Vaillancourt, "The far-infrared polarization spectrum: First results and analysis," ApJ 516, pp. 834-842, 1999.

14. A. Lazarian, A. A. Goodman, and P. C. Myers, "On the efficiency of grain alignment in dark clouds," ApJ 490, pp. 273-280, 1997.

15. A. Krabbe and S. C. Casey, "First Light SOFIA Instruments," in Infrared Spaceborne Remote Sensing X, M. Strojnik and B. F. Andresen, eds., Proc. SPIE 4818, pp. 1-13, 2002.

16. C. D. Dowell, J. A. Davidson, J. L. Dotson, R. H. Hildebrand, G. Novak, T. S. Rennick, and J. E. Vaillancourt, "Hale, a multi-wavelength, far-infrared polarimeter for SOFIA," in Polarimetry in Astronomy, S. Fineschi, ed., Proc. SPIE 4843, pp. 250-259, 2003.

17. C. C. Packham, D. Axon, J. H. Hough, T. J. Jones, P. F. Roche, C. M. Telesco, and M. Tamura, "Mid-IR polarimetry: new vistas for SOFIA," in this volume, Proc. SPIE 6678, 2007.

18. D. A. Harper, et al., "HAWC: A far-infrared camera for SOFIA," in Airborne Telescope Systems, R. K. Melugin and H.-P. Roeser, eds., Proc. SPIE 4014, pp. 43-53, 2000.

19. C. F. McKee and E. C. Ostriker, "Theory of Star Formation," ARA\&A 707, 2007. in press.

20. D. Biskamp, Magnetohydrodynamic Turbulence, Cambridge University Press, Cambridge, 2003.

21. J. Cho and A. Lazarian, "Generation of compressible modes in MHD turbulence," Theor. Comput. Fluid Dynamics 19, pp. 127-157, 2005.

22. T. J. Bethell, A. Chepurnov, A. Lazarian, and J. Kim, "Polarization of dust emission in clumpy molecular clouds and cores," ApJ 663, pp. 1055-1068, 2007.

23. A. Lazarian, "Tracing magnetic fields with aligned grains," J. Quant. Spectrosc. Radiat. Transfer 106, pp. 225-256, 2007.

24. A. Z. Dolginov and I. G. Mytrophanov, "Orientation of cosmic dust grains," Ap\&SS 43, p. 257, 1976.

25. B. T. Draine and J. C. Weingartner, "Radiative torques on interstellar grains. I. Superthermal spin-up," ApJ 470, pp. 551-565, 1996.

26. B. T. Draine and J. C. Weingartner, "Radiative torques on interstellar grains. II. Grain alignment," ApJ 480, pp. 633-646, 1997.

27. A. Lazarian and T. Hoang, "Radiative torques: analytical model and basic properties," MNRAS 378, pp. 910-946, 2007.

28. L. Spitzer, Jr. and T. A. McGlynn, "Disorientation of interstellar grains in suprathermal rotation," ApJ 231, pp. 417-424, 1979. 
29. E. M. Purcell, "Suprathermal rotation of interstellar grains," ApJ 231, pp. 404-416, 1979.

30. A. Lazarian, "Gold-type mechanisms of grain alignment," MNRAS 268, pp. 713-723, 1994.

31. A. Lazarian and W. G. Roberge, "Barnett relaxation in thermally rotating grains," ApJ 484, pp. 230-237, 1997.

32. A. Lazarian and B. T. Draine, "Nuclear Spin Relaxation within Interstellar Grains," ApJ 520, pp. L67-L70, 1999.

33. A. Lazarian and B. T. Draine, "Thermal Flipping and Thermal Trapping: New Elements in Grain Dynamics," ApJ 516, pp. L37-L40, 1999.

34. J. C. Weingartner and B. T. Draine, "Radiative torques on interstellar grains. III. Dynamics with thermal relaxation," ApJ 589, pp. 289-318, 2003.

35. T. Hoang and A. Lazarian, "Radiative torque alignment: Essential physical processes," MNRAS , 2007, in press.

36. D. Ward-Thompson, J. M. Kirk, R. M. Crutcher, J. S. Greaves, W. S. Holland, and P. André, "First observations of the magnetic field geometry in prestellar cores," ApJ 537, pp. L135-L138, 2000.

37. J. E. Vaillancourt, "Analysis of the far-infrared/submillimeter polarization spectrum based on temperature maps of Orion," ApJS 142, pp. 53-69, 2002.

38. J. E. Vaillancourt, "Polarized emission from interstellar dust," in Sky Polarisation at far-infrared to radio wavelengths: The Galactic Screen before the Cosmic Microwave Background, F. Boulanger and M.-A. Miville-Deschênes, eds., EAS Publ. Ser. 23, pp. 147-164, 2007.

39. J. E. Vaillancourt et al., "Submillimeter polarization spectrum of OMC-1 from SHARP." in prep.

40. J. Cho and A. Lazarian, "Grain alignment and polarized emission from magnetized T Tauri disks," ApJ , 2007, in press.

41. C. Dominik, J. Blum, J. N. Cuzzi, and G. Wurm, "Growth of dust as the initial step toward planet formation," Protostars 83 Planets V, pp. 783-800, 2007.

42. S. V. W. Beckwith and A. I. Sargent, "Particle emissivity in circumstellar disks," ApJ 381, pp. 250-258, 1991.

43. P. D'Alessio, N. Calvet, and L. Hartmann, "Accretion disks around young objects. III. Grain growth," ApJ 553, pp. 321-334, 2001.

44. J. Rodmann, T. Henning, C. J. Chandler, L. G. Mundy, and D. J. Wilner, "Large dust particles in disks around T Tauri stars," A\&\&A 446, pp. 211-221, 2006.

45. B. T. Draine, "On the submillimeter opacity of protoplanetary disks," ApJ 636, pp. 1114-1120, 2006.

46. M. Krejny, et al., "The variable-delay polarization modulator," in Millimeter and Submillimeter Detectors and Instrumentation for Astronomy III, J. Zmuidzinas, W. S. Holland, S. Withington, and W. D. Duncan, eds., Proc. SPIE 6275, p. 53, 2006.

47. P. J. Napier, "The EVLA project: Ten times more capability for the VLA," in Revealing the Molecular Universe: One Antenna is Never Enough, D. C. Backer, J. M. Moran, and J. L. Turner, eds., ASP Conf. Ser. 356, p. 65, 2006.

48. M. Tamura, J. H. Hough, J. S. Greaves, J.-I. Morino, A. Chrysostomou, W. S. Holland, and M. Momose, "First detection of submillimeter polarization from T Tauri stars," ApJ 525, pp. 832-836, 1999.

49. S. A. Balbus and J. F. Hawley, "A powerful local shear instability in weakly magnetized disks. I - Linear analysis.," ApJ 376, pp. 214-233, 1991.

50. T. C. Mouschovias and G. E. Ciolek, "Magnetic fields and star formation: A theory reaching adulthood," in The Origin of Stars and Planetary Systems, C. J. Lada and N. D. Kylafis, eds., NATO ASIC Proc. 540, p. 305, 1999.

51. M.-M. Mac Low and R. S. Klessen, "Control of star formation by supersonic turbulence," Rev. Mod. Phys. 76, pp. 125-194, 2004.

52. C. Heiles, "9286 stars: An agglomeration of stellar polarization catalogs," AJ 119, pp. 923-927, 2000.

53. A. Pereyra and A. M. Magalhães, "Polarimetry toward the IRAS Vela shell. II. Extinction and magnetic fields," ApJ 662, pp. 1014-1023, 2007.

54. C. R. Lawrence, "The Planck mission," in IR Space Telescopes and Instruments., J. C. Mather, ed., Proc. SPIE 4850, pp. 710-721, 2003. 
55. F. Yusef-Zadeh, M. Morris, and D. Chance, "Large, highly organized radio structures near the Galactic centre," Nature 310, pp. 557-561, 1984.

56. F. Yusef-Zadeh, J. W. Hewitt, and W. Cotton, "A 20 centimeter survey of the Galactic center region. I. Detection of numerous linear filaments," ApJS 155, pp. 421-550, 2004.

57. M. E. Nord, T. J. W. Lazio, N. E. Kassim, S. D. Hyman, T. N. LaRosa, C. L. Brogan, and N. Duric, "High-resolution, wide-field imaging of the Galactic center region at $330 \mathrm{MHz}, " A J$ 128, pp. 1646-1670, 2004.

58. G. Novak, et al., "First results from the submillimeter polarimeter for antarctic remote observations: Evidence of large-scale toroidal magnetic fields in the Galactic center," ApJ 583, pp. L83-L86, 2003.

59. D. P. Clemens, B. D. Kane, R. W. Leach, and R. Barvainis, "Millipol, a millimeter/submillimeter wavelength polarimeter - Instrument, operation, and calibration," PASP 102, pp. 1064-1076, 1990.

60. R. H. Hildebrand, J. A. Davidson, J. L. Dotson, C. D. Dowell, G. Novak, and J. E. Vaillancourt, "A primer on far-infrared polarimetry," PASP 112, pp. 1215-1235, 2000.

61. G. Siringo, E. Kreysa, L. A. Reichertz, and K. M. Menten, "A new polarimeter for (sub)millimeter bolometer arrays," A\&A 422, pp. 751-760, 2004.

62. W. D. Duncan, I. Robson, P. A. R. Ade, and S. E. Church, "Measurements of the submillimetre emission noise from Mauna Kea," in Multi-Feed Systems for Radio Telescopes, D. T. Emerson and J. M. Payne, eds., ASP Conf. Ser. 75, p. 295, 1995.

63. L. Nordh, G. Olofsson, O. Appelblad, and L. Klynning, "The far infrared transmission of crystal quartz at 3 K," Infrared Phys. 27, pp. 305-308, 1987.

64. F. Bréhat and B. Wyncke, "Measurement of the optical constant of crystal quartz at $10 \mathrm{~K}$ and $300 \mathrm{~K}$ in the far infrared spectral range: 10-600 $\mathrm{cm}^{-1}$," Int. J. Infrared \& Millimeter Waves 18, pp. 1663-1679, 1997.

65. D. P. Gonatas, X. D. Wu, G. Novak, and R. H. Hildebrand, "Systematic effects in the measurement of far-infrared linear polarization," Appl. Opt. 28, pp. 1000-1006, 1989.

66. D. T. Chuss, E. J. Wollack, S. H. Moseley, and G. Novak, "Interferometric polarization control," Appl. Opt. 45, pp. 5107-5117, 2006.

67. T. Manabe, J. Inatani, A. Murk, R. J. Wylde, M. Seta, and D. H. Martin, "A new configuration of polarization-rotating dual-beam interferometer for space use," IEEE Trans. Microwave Theory Tech. 51, 2003.

68. E. S. Battistelli, et al., "Far infrared polarimeter with very low instrumental polarization," in Polarimetry in Astronomy, S. Fineschi, ed., Proc. SPIE 4843, pp. 241-249, 2003.

69. D. T. Chuss, D. J. Benford, C. Walker, S. H. Moseley, G. Novak, J. G. Staguhn, and E. J. Wollack, "A 350- $\mu \mathrm{m}$ array polarimeter using translational modulators," in Ground-based Instrumentation for Astronomy, A. F. M. Moorwood and M. Iye, eds., Proc. SPIE 5492, pp. 1450-1460, 2004.

70. M. Houde, R. L. Akeson, J. E. Carlstrom, J. W. Lamb, D. A. Schleuning, and D. P. Woody, "Polarizing grids, their assemblies, and beams of radiation," PASP 113, pp. 622-638, 2001.

71. C. A. Allen, D. J. Benford, D. T. Chuss, T. M. Miller, S. H. Moseley, J. G. Staguhn, and E. J. Wollack, "Backshort-under-grid arrays for infrared astronomy," Nucl. Instrum. Meth. Phys. Res. 559, pp. 522-524, 2006.

72. W. Holland, et al., "SCUBA-2: a 10,000-pixel submillimeter camera for the James Clerk Maxwell Telescope," in Millimeter and Submillimeter Detectors and Instrumentation for Astronomy III, J. Zmuidzinas, W. S. Holland, S. Withington, and W. D. Duncan, eds., Proc. SPIE 6275, pp. 45-60, 2006.

73. K. D. Irwin, et al., "In-focal-plane squid multiplexer," Nucl. Instrum. Meth. Phys. Res. A 520, pp. 544-547, 2004.

74. M. D. Niemack, "Measuring two-millimeter radiation with a prototype multiplexed TES receiver for ACT," in Millimeter and Submillimeter Detectors and Instrumentation for Astronomy III, J. Zmuidzinas, W. S. Holland, S. Withington, and W. D. Duncan, eds., Proc. SPIE 6275, 2006.

75. J. G. Staguhn, et al., "GISMO: a 2-millimeter bolometer camera for the IRAM $30 \mathrm{~m}$ telescope," in Millimeter and Submillimeter Detectors and Instrumentation for Astronomy III, J. Zmuidzinas, W. S. Holland, S. Withington, and W. D. Duncan, eds., Proc. SPIE 6275, 2006. 